Case report

\title{
Insulin autoimmune syndrome possibly caused by coenzyme Q10
}

\author{
Yoshiro Kusano ${ }^{1}$ \\ ${ }^{1}$ Third Department of Internal Medicine, Shirakawa Kosei General Hospital, Japan
}

\begin{abstract}
A 52-year-old woman was transported for reduced consciousness. Her blood glucose was only $19 \mathrm{mg} / \mathrm{dL}$, but her blood immunoreactive insulin and insulin antibody levels were high at $250 \mu \mathrm{U} / \mathrm{mL}$ and $50 \mathrm{U} / \mathrm{mL}$, respectively. She had no history of insulin treatment, but she had been taking coenzyme Q10 supplements for three months. Her human leukocyte antigen serotype was DR4. After stopping coenzyme Q10, her hypoglycemia disappeared and immunoreactive insulin and insulin antibody levels normalized. Based on the above, she was diagnosed with insulin autoimmune syndrome caused by coenzyme Q10. It is necessary to be aware of the onset of insulin autoimmune syndrome due to coenzyme Q10. Its pathogenesis requires clarification.
\end{abstract}

Key words: insulin autoimmune syndrome, hypoglycemia, coenzyme Q10

(J Rural Med 2019; 14(1): 132-137)

\section{Introduction}

Insulin autoimmune syndrome (IAS) is characterized by spontaneous hypoglycemia without evidence of exogenous insulin administration. IAS has a high serum concentration of total immunoreactive insulin (IRI) and high insulin autoantibody titerl). IAS has been associated with drugs or supplements containing sulfhydryl compounds in $52 \%$ of cases $^{2,3)}$. I experienced a case of IAS due to coenzyme Q10 (CoQ10) without sulfhydryl compounds and report this side

Received: May 15, 2018

Accepted: December 21, 2018

Correspondence: Yoshiro Kusano, Third Department of Internal Medicine, Shirakawa Kosei General Hospital, Yajirou, Toyochiue, Shirakawa-shi, Fushima 961-0005, Japan

E-mail: yo55@coral.ocn.ne.jp

This is an open-access article distributed under the terms of the Creative Commons Attribution Non-Commercial No Derivatives (by-nc-nd) License $<$ http://creativecommons.org/licenses/by-nc-nd/4.0/>. effect of CoQ10.

IRI levels were measured by chemiluminescence immunoassay using ARCHITECT insulin (Abbott, Tokyo, Japan). Serum insulin antibody levels were measured by radioimmunoassay using insulin antibody (Cosmic, Tokyo, Japan).

\section{Case Report}

Case: A 52-year-old woman

Chief complaint: Sweating and weakness

Family history: Unremarkable

Medical history: At the age of 27 years, the patient developed toxemia during her third pregnancy. Afterward, her blood pressure remained high despite taking antihypertensive medication. She had no history of diabetes, insulin use, or sulfonylurea (SU) drugs. She had been taking loxoprofen for headaches since she was 42 years old. At the age of 52 years, she was taking olmesartan $40 \mathrm{mg}$ / day, azelnidipine $8 \mathrm{mg} /$ day, lansoprazole $15 \mathrm{mg} /$ day, loxoprofen $60 \mathrm{mg} / \mathrm{day}$, and zolpidem tartrate $5 \mathrm{mg} /$ day. She had started taking a CoQ10 supplement three months prior to her admission. She had recently started to sweat frequently. One evening, she experienced profuse sweating, and her consciousness declined. She was admitted to the emergency department. At that time, she was in a coma and her blood glucose level was $19 \mathrm{mg} / \mathrm{dL}$. Following intravenous glucose injection, her blood glucose level increased to $129 \mathrm{mg} / \mathrm{dL}$ and she recovered consciousness. She was hospitalized for examination.

Physical findings: Height, $157 \mathrm{~cm}$; weight, $56 \mathrm{~kg}$; body mass index, $23 \mathrm{~kg} / \mathrm{m}^{2}$; body temperature, $34.3^{\circ} \mathrm{C}$; blood pressure, 207/96 $\mathrm{mmHg}$; and pulse rate, $60 / \mathrm{min}$. Her consciousness was clear. Chest auscultation revealed no heart murmur. Her abdomen was flat and without tenderness. In chest radiograph, heart enlargement with a cardiothoracic ratio of $58 \%$ and left ventricular hypertrophy of sV1 $+\mathrm{rV} 6=$ $4.6 \mathrm{mV}$ by electrocardiography (ECG) were observed. The 
Table 1 Laboratory data on admission

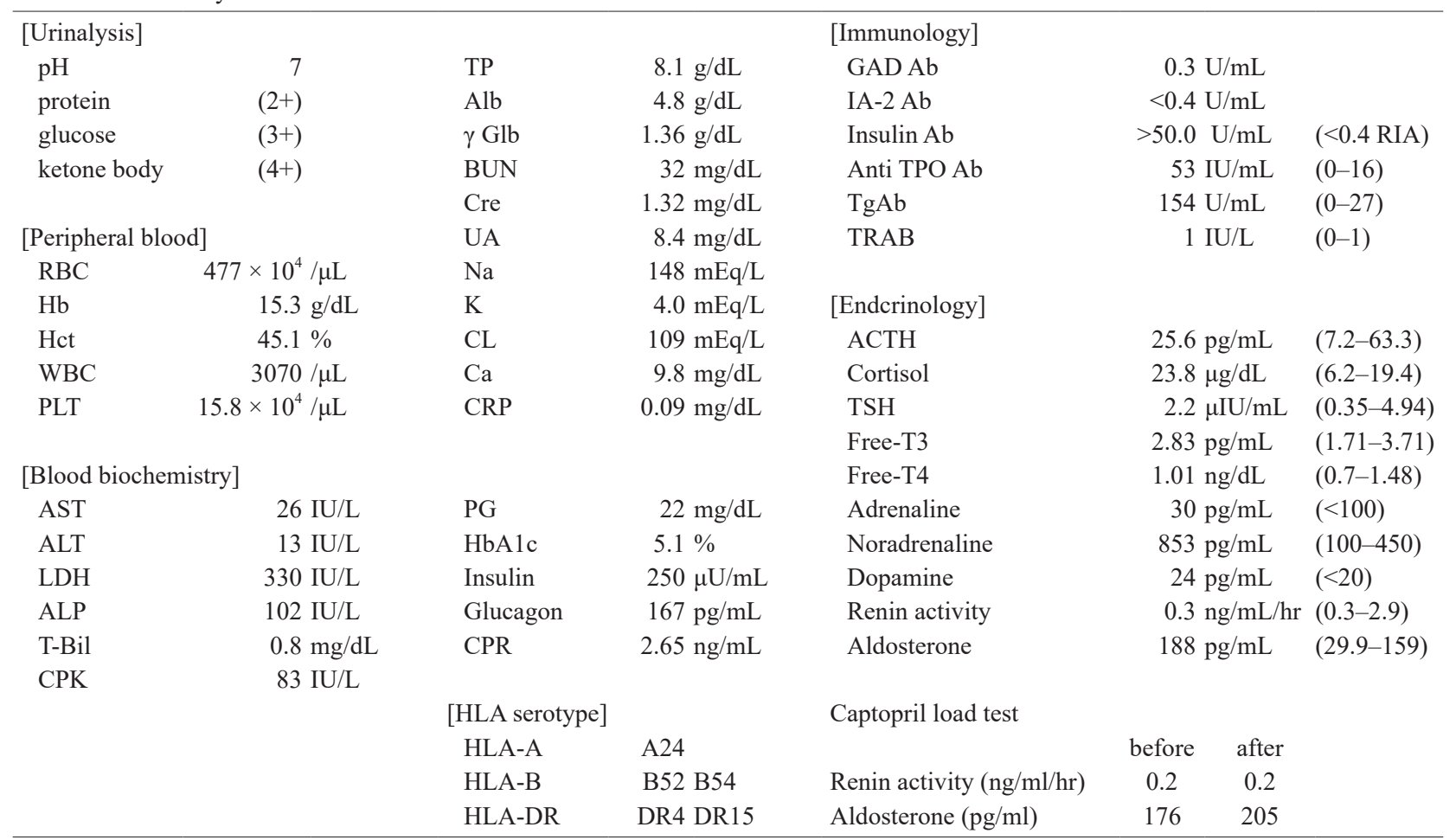

thyroid gland was not enlarged.

Blood examination: Her blood glucose level was as low as $22 \mathrm{mg} / \mathrm{dL}$ and her glycated hemoglobin (HbAlc) level was normal, at $5.1 \%$. Her blood IRI level was as high as $250 \mu \mathrm{U} / \mathrm{mL}$, and her serum C-peptide level was also high, at $2.65 \mathrm{ng} / \mathrm{mL}$. Her insulin antibody level was high, at 50 $\mathrm{U} / \mathrm{mL}$ or greater. She tested negative for anti-glutamic acid decarboxylase (anti-GAD), anti-tyrosine phosphatase-like insulinoma antigen 2 (anti-IA-2), and antinuclear antibodies. Anti-thyroglobulin antibody $(\mathrm{TgA} b)$ and anti-thyroperoxidase antibody (TPOAb) levels were high, but the thyroid receptor antibody (TRAB) level was normal. Serum creatinine was as high as $1.32 \mathrm{mg} / \mathrm{dL}$, and the levels of blood urea nitrogen, uric acid, and serum Na were also high. She was positive for urinary protein. Endocrine tests revealed high plasma cortisol, noradrenaline, and aldosterone levels and low plasma renin activity (Table 1).

After hospitalization, IAS was suspected due to her high IRI and insulin antibody levels. Since IAS is often caused by drugs, CoQ10 and loxoprofen were discontinued and olmesartan was changed to telmisartan $80 \mathrm{mg} /$ day, azelnidipine was changed to amlodipine $10 \mathrm{mg} /$ day, and the administration of lansoprazole was continued. She became hypoglycemic several times a day until the third day after hospitalization, when she received intravenous injections $(40 \mathrm{~mL})$ of $50 \%$ glucose. Her hypoglycemia decreased. The evaluation of diurnal variations showed that her blood glucose level was within 67 to $239 \mathrm{mg} / \mathrm{dL}$ but her IRI was very high at 240 to $1,258 \mu \mathrm{U} / \mathrm{mL}$ (Figure 1a). A 75 -g glucose tolerance test (GTT) revealed boundary-type blood glucose level and her IRI had an over-delayed response (Fig. 1b). Continuous glucose monitoring (CGM) 16 days after hospitalization showed hypoglycemia after midnight and at around noon (Figure 1c). The hypoglycemia disappeared after 17 days of hospitalization. Abdominal dynamic computed tomography and magnetic resonance imaging showed no tumors suggestive of insulinoma in the pancreas or other organs but a nodule-like shadow was observed in the left adrenal gland (Figure 2a, 2b). In this case, the insulin antibody titer was high and IAS was suspected, so fasting tests were not conducted.

A Scatchard plot analysis of the insulin antibody (requested by SRL, Inc.) showed two classes of binding sites. One site had an affinity constant (K1) of $0.0321 \times 10^{8} \mathrm{M}^{-1}$ with $29.1 \times 10^{-8} \mathrm{M}$ (high-affinity/low binding site) binding sites (R1). The other had an affinity constant (K2) of $0.000920 \times 10^{8} \mathrm{M}^{-1}$ and $102 \times 10^{-8} \mathrm{M}$ (low-affinity/high binding site) binding sites (R2) (Figure 3). The Scatchard plot analysis in this case was measured using porcine insulin. Porcine and human insulin differ in one amino acid sequence. Thus, the analysis in this case may not be accurate, but is rather a reference value. 
a
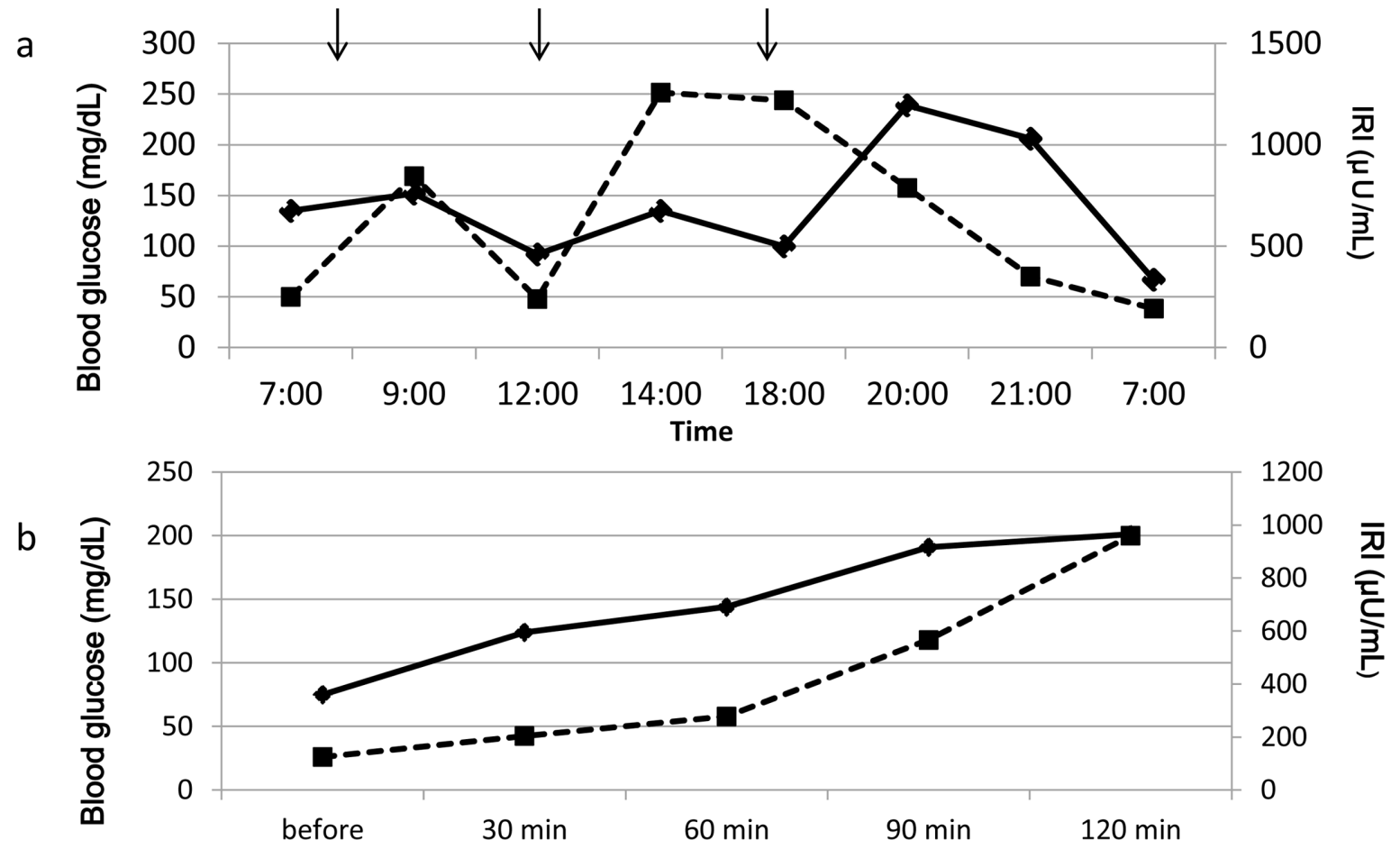

C

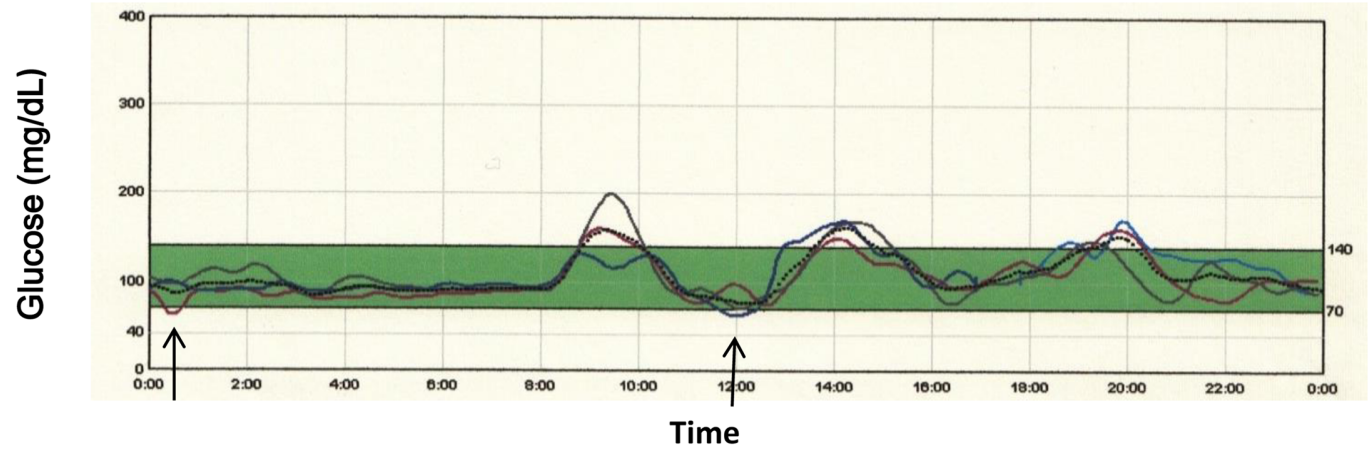

Figure 1 Diurnal variation of blood glucose and IRI levels, 75-g GTT, and CGM. IRI: immunoreactive insulin; CGM: Continuous glucose monitoring system (iPro ${ }^{\mathrm{TM}} 2$; Medtronic). a. Diurnal variations in blood glucose and IRI; solid line: blood glucose, dotted line: IRI, arrow: meal. b. 75-g GTT; solid line: blood glucose, dotted line: IRI. c. CGM; Red line: 15 days after hospitalization. Black line: 16 days after hospitalization. Blue line: 17 days after hospitalization. Arrow: hypoglycemia.

The patient's HLA serotype was DR4 (Table 1). HLA DNA typing was not performed.

Her blood pressure was as high as $150-180 / 80-106$ $\mathrm{mmHg}$ and her plasma aldosterone level was high. In the captopril load test, the aldosterone-renin ratio (ARR) one hour after loading was as high as 1025 , suggestive of primary aldosteronism (PA) (Table 1). The patient refused surgery and was discharged after her blood pressure declined due to the addition of antihypertensive medicine.

\section{Post-discharge course}

The patient had no recurrence of hypoglycemia. Her IRI and insulin antibody levels decreased to the normal ranges after 9 months (Figure 4).

\section{Discussion}

Here, I report a case of IAS possibly caused by CoQ10. Half of the IAS cases occur naturally, and the others occur due to drugs ${ }^{3}$. Many drugs that cause IAS contain sulfhydryl (SH) groups in their molecular structure (i.e., thiamazole, 

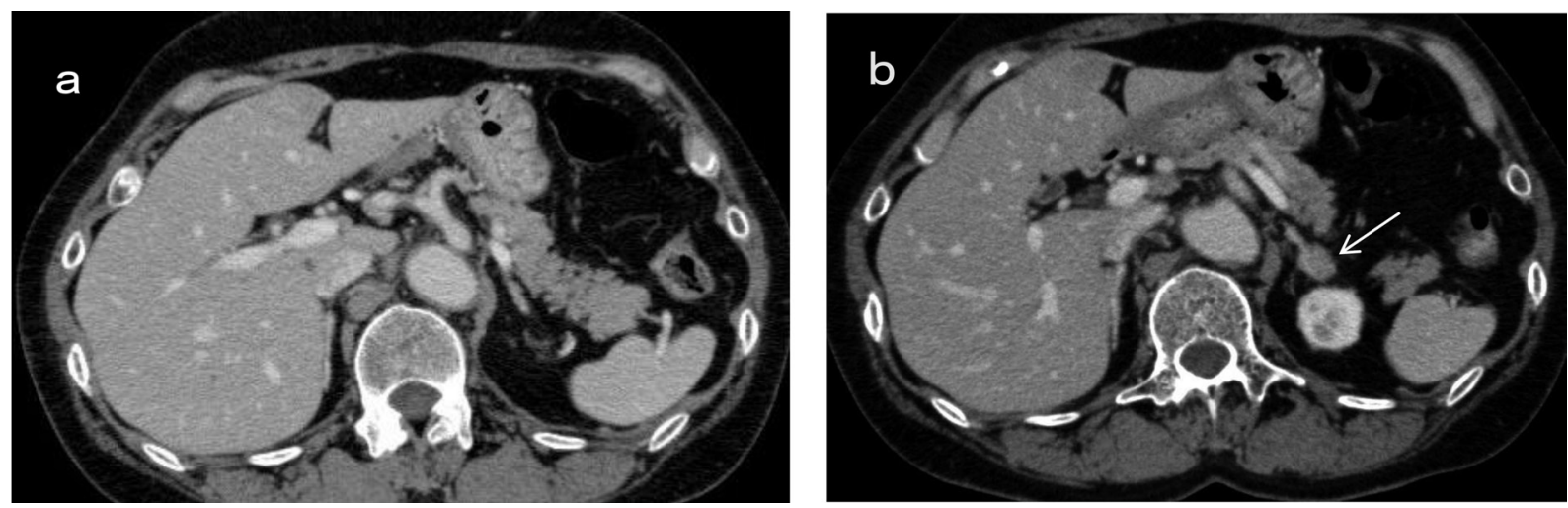

Figure 2 Abdominal computed tomography findings. a. No tumor is visible in the pancreas. b. A nodule is visible in the left adrenal gland (arrow).

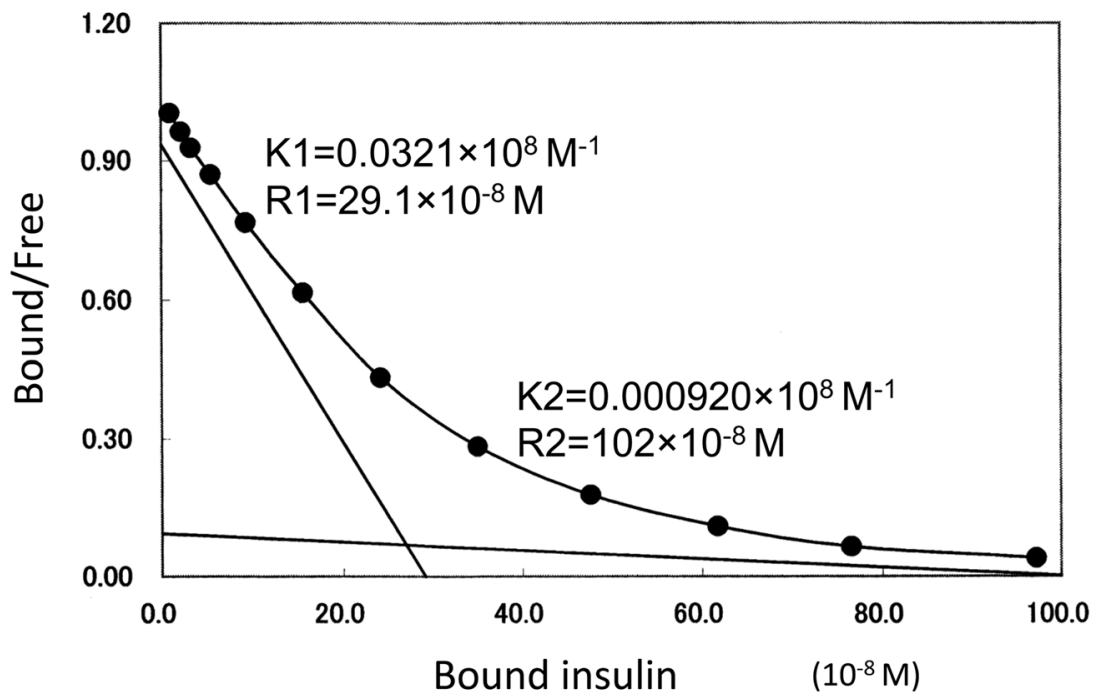

Figure 3 Scatchard plot analysis of insulin antibody levels. Affinity constant at high-affinity sites: K1. Low-affinity site: K2. Number of high-affinity and low-affinity binding sites R1 and R2, respectively. Values were measured by RIA using porcine insulin.

tiopronin, glutathione, bucillamine, and D-penicillamine) $)^{2,3)}$. Thus, supplements containing $\alpha$-lipoic acid or clopidogrel can cause IAS because their metabolites contain $\mathrm{SH}$ groups ${ }^{4,5}$. The SH group activates T-cells by cleaving the S-S bond of insulin to produce an antibody against insulin ${ }^{6,7)}$. Additionally, loxoprofen without $\mathrm{SH}$ also reportedly causes IAS $^{8}$. In IAS caused by drugs, hypoglycemia occurs $4-6$ weeks after drug ingestion". In the present case, the patient was not taking drugs with SH groups, but had started taking CoQ10 three months before her hypoglycemia. After stopping CoQ10 supplementation, her hypoglycemia disappeared and the insulin antibody level decreased. Therefore, her IAS was likely caused by CoQ10. Loxoprofen is unlikely to cause IAS because she had taken it for headaches for 10 years, during which time the IAS was not noted. In a nationwide survey of spontaneous hypoglycemia in Japan in 2009, CoQ was associated with $5 \%$ of the cases of IAS ${ }^{2}$. However, a search of Igaku Chuo Zasshi and PubMed revealed no case reports of IAS due to CoQ.

CoQ10 has an antioxidant effect and protects cells from oxidation by scavenging free radicals. Therefore, exogenous CoQ10 supplements may improve oxidative stress-induced abnormalities in mitochondrial functions ${ }^{10}$. CoQ10 does not have an SH group and the mechanism that causes IAS is currently unknown.

Insulin antibodies are often observed in patients undergoing insulin treatment. These antibodies usually do not cause hypoglycemia as they almost never bind to insulin 


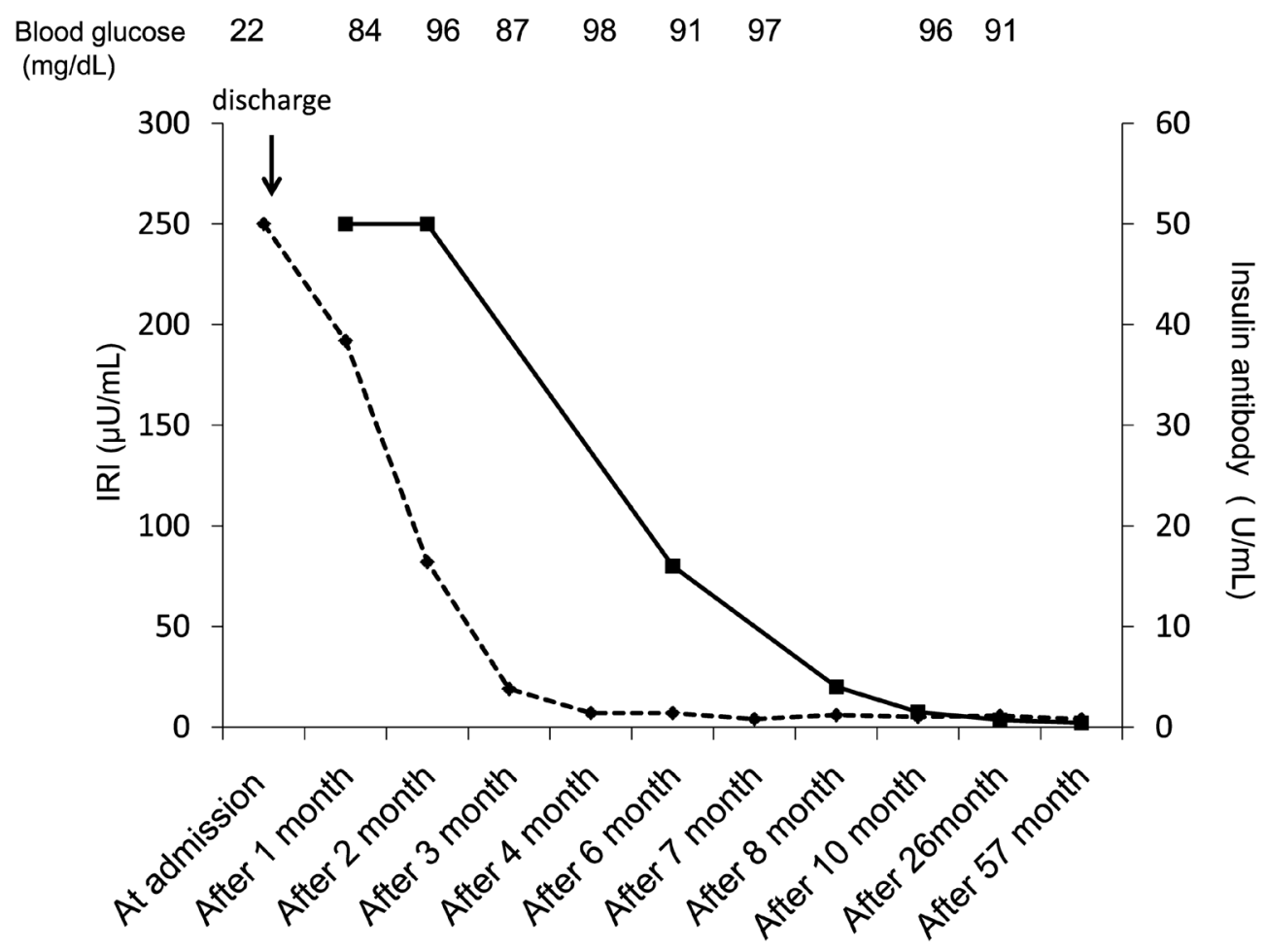

Figure 4 The patient's clinical course. Hyperinsulinemia and insulin antibody levels improved after discharge (fasting). dotted line: IRI, solid line: insulin antibody.

(binding capacities: $0.18-1.10 \times 10^{-8} \mathrm{M}$ ). If they do bind to insulin, they never separate (high-affinity constants: 4.02-7.11 $\left.\times 10^{8} \mathrm{M}^{-1}\right)^{11}$. However, the insulin antibodies in IAS have larger binding capacities $\left(11.5-55.4 \times 10^{-8} \mathrm{M}\right)$ and lower affinity constants $\left(0.021-0.24 \times 10^{8} \mathrm{M}^{-1}\right)$ than those of insulin antibodies in insulin-treated cases ${ }^{11}$. Therefore, insulin antibodies in IAS easily bind and dissociate from insulin, inducing hypoglycemia. The insulin antibodies in this case had characteristics of IAS antibodies.

There is a strong association between HLA alleles and IAS. Japanese patients with IAS are DR4-positive in $96 \%$ of cases and many are DRB1*0406-positive ${ }^{12,13)}$. The present patient had DR4, but HLA typing was not performed.

IAS merges autoimmune diseases, such as Graves' disease and others ${ }^{14,15)}$. In this case, the levels of TPOAb and $\mathrm{TgAb}$ were high. Therefore, the patient also had Hashimoto's thyroiditis and immune abnormalities.

Furthermore, the patient also had PA. The PA may have been present at 27 years of age when she was diagnosed with essential hypertension. She had been treated with oral antihypertensive drugs for a long time. PA and IAS are assumed to be coincident because aldosterone does not affect the immune system.

I experienced a case of IAS possibly caused by CoQ10.
CoQ10 is marketed as a supplement and many people use it. Attention should be paid to the potential for the onset of IAS by CoQ10. It is necessary to determine the mechanism of its pathogenesis.

Conflict of interest: The author states no conflict of interest.

\section{References}

1. Hirata $\mathrm{Y}$, Ishizu $\mathrm{H}$, Ouchi $\mathrm{N}$, et al. Insulin autoimmunity in a case of spontaneous hypoglycemia. Journal of the Japan Diabetes Society. 1970; 13: 312-320 (in Japanese).

2. Uchigata Y, Hirata Y, Iwamoto Y. Drug-induced insulin autoimmune syndrome. Diabetes Res Clin Pract 2009; 83: e19e20. [Medline] [CrossRef]

3. Uchigata Y. New findings of insulin autoimmune syndrome. Journal of the Japan Diabetes Society. 2011; 54: 877-879 (in Japanese).

4. Moffa S, Improta I, Rocchetti S, et al. Potential cause-effect relationship between insulin autoimmune syndrome and alpha lipoic acid: Two case reports. Nutrition 2019; 57: 1-4. [Medline] [CrossRef]

5. Rajpal A, Kassem LS, Moscoso-Cordero M, et al. Clopidogrel-induced insulin autoimmune syndrome: a newly recog- 
nized cause of hypoglycemia in a patient without diabetes. $\mathrm{J}$ Endocr Soc 2017; 1: 1217-1223. [Medline] [CrossRef]

6. Dozio N, Scavini M, Beretta A, et al. Imaging of the buffering effect of insulin antibodies in the autoimmune hypoglycemic syndrome. J Clin Endocrinol Metab 1998; 83: 643-648. [Medline]

7. Matsushita S, Takahashi K, Motoki M, et al. Allele specificity of structural requirement for peptides bound to HLADRB $1 * 0405$ and DRB $1 * 0406$ complexes: implication for the HLA-associated susceptibility to methimazole-induced insulin autoimmune syndrome. J Exp Med 1994; 180: 873-883. [Medline] [CrossRef]

8. Okazaki-Sakai S, Yoshimoto S, Yagi K, et al. Insulin autoimmune syndrome caused by an adhesive skin patch containing loxoprofen-sodium. Intern Med 2013; 52: 2447-2451. [Medline] [CrossRef]

9. Hirata Y. Methimazole and insulin autoimmune syndrome with hypoglycemia. Lancet 1983; 2: 1037-1038. [Medline] [CrossRef]

10. Bhagavan HN, Chopra RK. Coenzyme Q10: absorption, tis- sue uptake, metabolism and pharmacokinetics. Free Radic Res 2006; 40: 445-453. [Medline] [CrossRef]

11. Eguchi Y. Scatchard analysis of insulin autoantibodies in the insulin autoimmune syndrome. Journal of Tokyo Women's Medical University 1998; 59: 1296-1305 (in Japanese, Abstract in English).

12. Uchigata Y, Kuwata S, Tokunaga K, et al. Strong association of insulin autoimmune syndrome with HLA-DR4. Lancet 1992; 339: 393-394. [Medline] [CrossRef]

13. Uchigata Y, Hirata Y, Omori Y, et al. Worldwide differences in the incidence of insulin autoimmune syndrome (Hirata disease) with respect to the evolution of HLA-DR4 alleles. Hum Immunol 2000; 61: 154-157. [Medline] [CrossRef]

14. Torimoto K, Okada Y, Mori H, et al. Two sisters with Graves' Disease and similar clinical features who tested positive for anti-insulin antibodies after thiamazole treatment. Intern Med 2016; 55: 1125-1129. [Medline] [CrossRef]

15. Rouabhia S, Ramanoelina J, Godmer P, et al. Insulin autoimmune syndrome revealing systemic lupus erythematosus. Ann Med Interne (Paris) 2003; 154: 59-60 (in French). [Medline] 